\title{
PERFORMANCE ANALYSIS OF LINEAR FRESNEL REFLECTOR CONCENTRATING SOLAR SYSTEM WITH HORIZONTAL AND VERTICAL ABSORBER WITH VARYING WIDTH OF REFLECTOR
}

\author{
M.Babu ${ }^{1}$, S.Hariharan ${ }^{2}$ and T. Pushparaj ${ }^{3}$
}

\begin{abstract}
In this paper, the effect of reflector width on the performance of Linear Fresnel Reflector (LFR) concepts with horizontal and vertical absorber is examined. A theoretical model encompassing all the governing parameters is developed by taking into account the intricate phenomenal interdependencies and influences of parameters on the designs output. The designed and subsequently realized test LFR system with horizontal and vertical absorbers are evaluated major from the insight of concentration ratio. Two systems fitted with horizontal and vertical absorbers with reflectors of variable width arrangements have been investigated. The parameters for these two systems are derived from their geometrical representation. Further, effect of different design parameters on the concentration ratio for both the systems is thoroughly investigated. Experiments and their performance are evaluated based on the concentration ratio. The design procedures and the characterization methods have been summarized. The formulae being iterative in nature are solved computationally using programs written in MATLAB. The formulae are unable to yield a single solution; instead of they give a set of solutions when each of the system parameters is width of reflector plates, number of reflector plates, land area constraints is varied. Optimal design parameters are selected from the solutions which in turn minimizes the material and land requirements. This procedure is repeated for the two different configurations and thermal analysis is performed in terms of concentration ratio. Optimal design parameters are selected by computing and plotted in graph. The distribution of Local Concentration Ratio (LCR) on the surface of the horizontal and vertical absorbers for both the designs is investigated using analytical technique. The angular error is modelled using Gaussian distribution and its impact on the LCR is also analysed. The final observations are recorded and their outcome emphasizes that the horizontal absorber with varying width outperforms the system with vertical absorber with varying reflector width.
\end{abstract}

Keywords: Linear Fresnel reflector, Horizontal absorber, Vertical absorber, Reflector width, Concentration ratio, Concentrated Power, Orientation error.

\section{INTRODUCTION}

Linear Fresnel Reflector (LFR) is a line-focusing system. A typical LFR uses modular, flat mirrors that track the sun to focus the sun's heat onto long, elevated tubular receiver (absorber) through which water flows. The concentrated sunlight boils the water in the tubes, generating hot water, saturated or superheated steam for use in power generation in steam engine or turbine plant works with the Rankine cycle for instance or for process heat in industrial applications. The Rankine cycle is an idealized thermodynamic cycle of a heat engine that converts heat into mechanical work. The heat is supplied externally to a closed loop, which usually uses water as the working

\footnotetext{
${ }^{1}$ Department of Mechanical Engineering Kings College of Engineering, Pudukkottai - 613303, Tamil Nadu, India

${ }^{2}$ Department of Mechanical Engineering Kings College of Engineering, Pudukkottai - 613303, Tamil Nadu, India

${ }^{3}$ Department of Mechanical Engineering Kings College of Engineering, Pudukkottai - 613303, Tamil Nadu, India
} 
fluid. The Rankine cycle, in the form of steam engines, generates about $90 \%$ of all electric power used throughout the world. The efficiency of the Rankine cycle is limited by the high heat of vaporization of the working fluid. The Rankine cycle used with solar energy is known as the organic Rankine cycle solar power plant. The Organic Rankine Cycle (ORC) power plant is much simpler than a conventional steam Rankine cycle power plant and allows unattended operation of the facility [1]. It was further observed that the efficiency of the system fluctuates significantly and is dependent on global radiation. Michael J Wagner [2] presented an overview of the recently added LFR model. Besides it reports a Levelized Cost of Energy LCOE-optimized plant comparison between various Concentrating Solar Power technologies. The analysis reveals that while LFR is cost-competitive with other Concentrated Solar Power (CSP) technologies -especially Parabolic Troughs. The point of stress was the numerous advantages offered by LFR, including low-cost collectors and simplified receiver piping that facilitate relatively high-temperature operation. [3] Reynolds presented "oven-box" concept that employs a set of small parallel absorber tubes nested within a trapezoidal insulated cavity. [4] Gabriel Morin developed the compound parabolic secondary concentrator tubular receiver and analysed its performance and techno-economical factors. A comparative study was undertaken for evaluating the thermal performance of the four identical trapezoidal cavity absorbers for linear Fresnel reflecting solar device by Panna Lal Singh [5] and Baum [6] studied two-axis solar tracking systems. However, the pivotal attempt to employ it for large linear system for solar collection was developed both linear and two- axis tracking Fresnel reflector systems [7]. [8] Riaz developed the associated theory for two-axis systems. [9] Mathur presented elaborate information on optical designs of LFR solar concentrator with three different geometries: horizontal, flat vertical and tubular receivers. Concentration ratio was determined using analytical and ray trace technique. [10] Martin Haagen presented the estimation of the prospective of generating solar heat for industrial processes with linear Fresnel collectors. A single variable sensitivity analysis examines the factors which influence the internal rate of return and thermal energy costs of solar process heat systems.[11] Manikumar performed numerical simulation of a trapezoidal cavity absorber in the linear Fresnel reflector solar concentrator system. Different approaches were employed for designing a LFRSC. Concentration of solar radiation is necessary for efficient solar thermal and photovoltaic systems Duffle [12] and Harry [13]. Designs have been developed to utilize line focus solar concentrators for reducing the cost of the present systems Harry [13] and Mathur [14]. [15] Marchi LFR is the lowest cost option for large scale solar electricity plant in the foreseeable future David [16] detailed performance analysis needs to be done for better efficiency, cost effective, suitability and efficiently use the solar radiation. Thus, analysis should be investigated for the distribution of local concentration ratio (LCR). The assumptions are made to facilitate the designing of Linear Fresnel Reflector Solar Concentrator (LFRSC) (i) The reflector elements are specularly reflective and (ii) the solar radiation incident normally on the reflector elements [17]. (i) The concentrator is perfectly tracked so as to follow the diurnal movement of the sun and (ii) The solar radiation is axially incident [18]. The same assumptions are followed in this paper.

In the present work a detailed evaluation of two designs of Linear Fresnel Reflector Concentrating Solar (LFRCS) system with horizontal absorber and vertical absorber has been undertaken. In such concentrating system long narrow plane mirror are mounted on flat base [19]. The mirror elements are tilted at an angle so that the solar radiation incident normally on any mirror element, after reflection, illuminates a common focus. LFRCS system can be designed using two different approaches. In one approach, using the pre-specified size of absorber, the size of mirror element and hence the size of the concentrator are determined [19]. This approach allows a variation in the width of the constituent mirror elements. In the second approach using an equal width of the constituent mirror element, the size of absorber is determined. The design of a LFRSC with flat vertical absorber has shown that a more or less uniform illumination can be obtained on both the surface of the absorber for pre-specified width. The approach suggested for obtaining uniform illumination is to allow a variation in the width of the constituent mirror element so that for normally incident solar radiation the width of the image produced on the absorber by each mirror element matches with the pre-specified width of the absorber [20]. A comparative study between the LFRCS with horizontal and vertical absorber system has to be carried out. The detailed design and performance characteristics of the LFRSC employing horizontal absorber and vertical absorber with varying width are described in the present paper and the distribution of local concentration ratio (LCR) on the absorber surface has been determined for the each design using the analytical technique. The formulas for the various parameters have been derived from the geometrical figures plotted from the system design according to the absorber orientation based on the two systems horizontal absorber and vertical absorber.

\section{DESIGN OF LFRCS SYSTEM WITH HORIZONTAL AND VERTICAL ABSORBER WITH VARYING REFLECTOR WIDTH}


Figure 1 and 2 represents arrangement of LFRCS system design with horizontal and vertical absorbers configuration with varying reflector width. Figure 1 and 2 shows the geometry of a LFR with reflecting elements of varying width, which is designed using conventional geometrical optics. In the present case, the aperture diameter, $D$, of the concentrator is fixed and the required number of reflecting elements is determined by optical considerations. The first reflecting element should be placed at an appropriate location $\left(Q_{1}\right)$, so it can contribute to the concentration on the absorber surface. An iterative approach along with simple geometrical optics is incorporated to determine the design parameters of the reflecting elements. The initial values for location, tilt, and shift are set as $Q_{0}=d / 2, \theta_{0}=0$ and $S_{1}=0$ and $W_{0}=0$ respectively, where $d$ is the absorber width. The initial tilt $\theta_{n}$, for all the " $n$ " reflecting element is set as zero " 0 " [18]. The angular sub-tense of the sun at any point on the earth (=32') has been taken into account while designing the concentrators. The generalized formula for the various design parameters have been derived for each design.

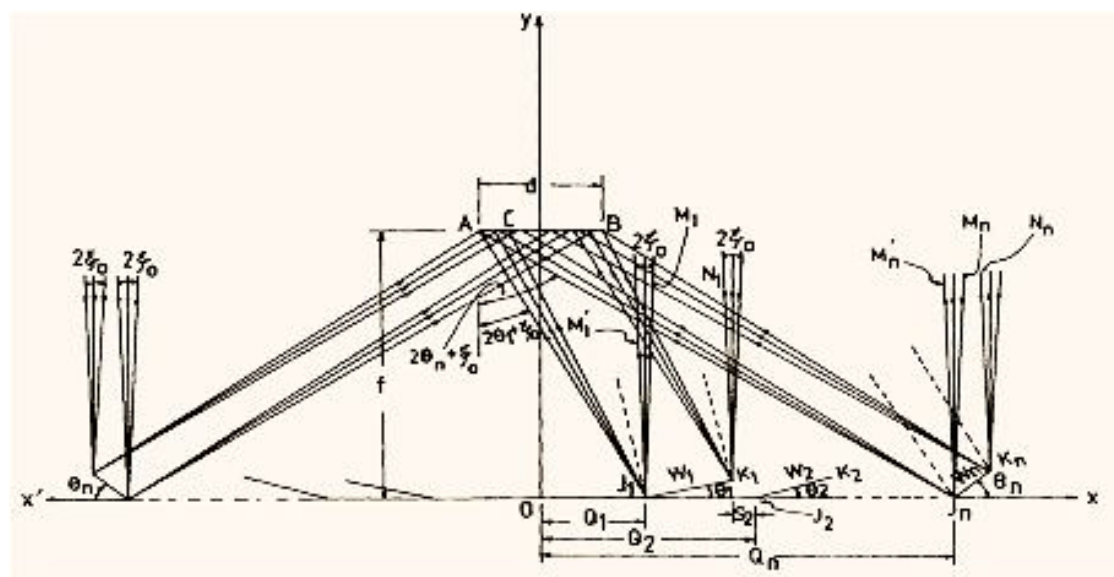

Figure 1: Linear Fresnel reflector concentrating solar (LFRCS) system horizontal absorber with varying width reflector.

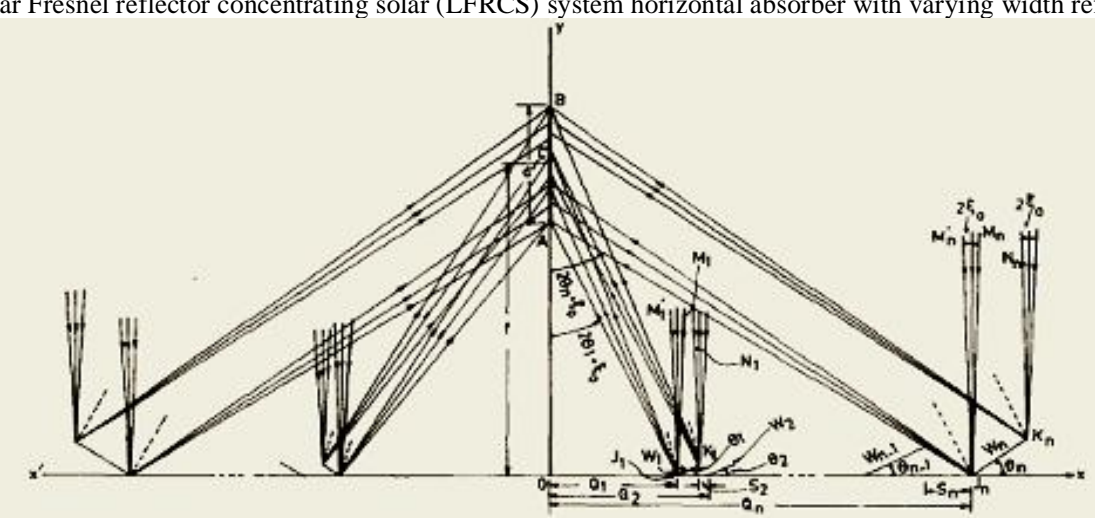

Figure 2: Linear Fresnel reflector concentrating solar LFRCS system vertical absorber with varying width reflector

\section{DERIVATION OF HORIZONTAL ABSORBER WITH VARYING WIDTH REFLECTOR LFR SYSTEM CONDITIONS}

- The reflected ray from the bottom of the reflector plate should reach the focal plane at $-d / 2$ and the reflected ray from the top of the plate should reach at $+d / 2$.

- The reflected ray from the bottom of $\mathrm{n}^{\text {th }}$ reflector should graze the top of $(\mathrm{n}-1)^{\text {th }}$ reflector.

The two triangles $\mathrm{CAB}$ and $\mathrm{BPQ}$ are similar triangles and are used to derive the relation for shift between subsequent plates, from Figure 3 (a).

Equating the base and height ratios of the two triangles,

$S_{n}\left(\mathrm{~W}_{\mathrm{n}-1} \cdot \sin \theta_{\mathrm{n}-1}\right)=\left(Q_{n-1}+\mathrm{W}_{\mathrm{n}-1} \cdot \cos \theta_{\mathrm{n}-1}+\mathrm{d} / 2\right) /\left(f-\mathrm{W}_{\mathrm{n}-1} \cdot \sin \theta_{\mathrm{n}-1}\right)$

In triangle CSQ,

$\tan \left(2 \theta_{\mathrm{n}}+\xi_{0}\right)=\left[\left(Q_{n}+d / 2\right) f\right]$ 
Performance Analysis Of Linear Fresnel Reflector Concentrating Solar System With Horizontal And Vertical Absorber With Varying Width Of Reflector

For formulating the width of the reflector plate we equalize the image width to the width of the absorber, from Figure 3 (b)

Image width $=$ absorber width

$\mathrm{d}=\left(\mathrm{U}_{\mathrm{n}}+\mathrm{J}_{\mathrm{n}}+\mathrm{I}_{\mathrm{n}}\right)$

$\mathrm{U}_{\mathrm{n}}=\frac{\left(f-W_{n} \cdot \sin \theta_{\mathrm{n}}\right) \sec 2 \theta_{\mathrm{n}} \cdot \sin \xi_{0}}{\left[\cos \left(2 \theta_{\mathrm{n}}-\xi_{0}\right)\right]}$

$J_{n}=\left[W_{n} \cos \theta_{n} \cdot \sec 2 \theta_{\mathrm{n}}\right]$

$\mathrm{I}_{\mathrm{n}}=\frac{[f \cdot \sec 2 \theta \cdot \sin \xi]}{\left[\cos \left(2 \theta_{0}+\xi_{0}\right)\right]}$
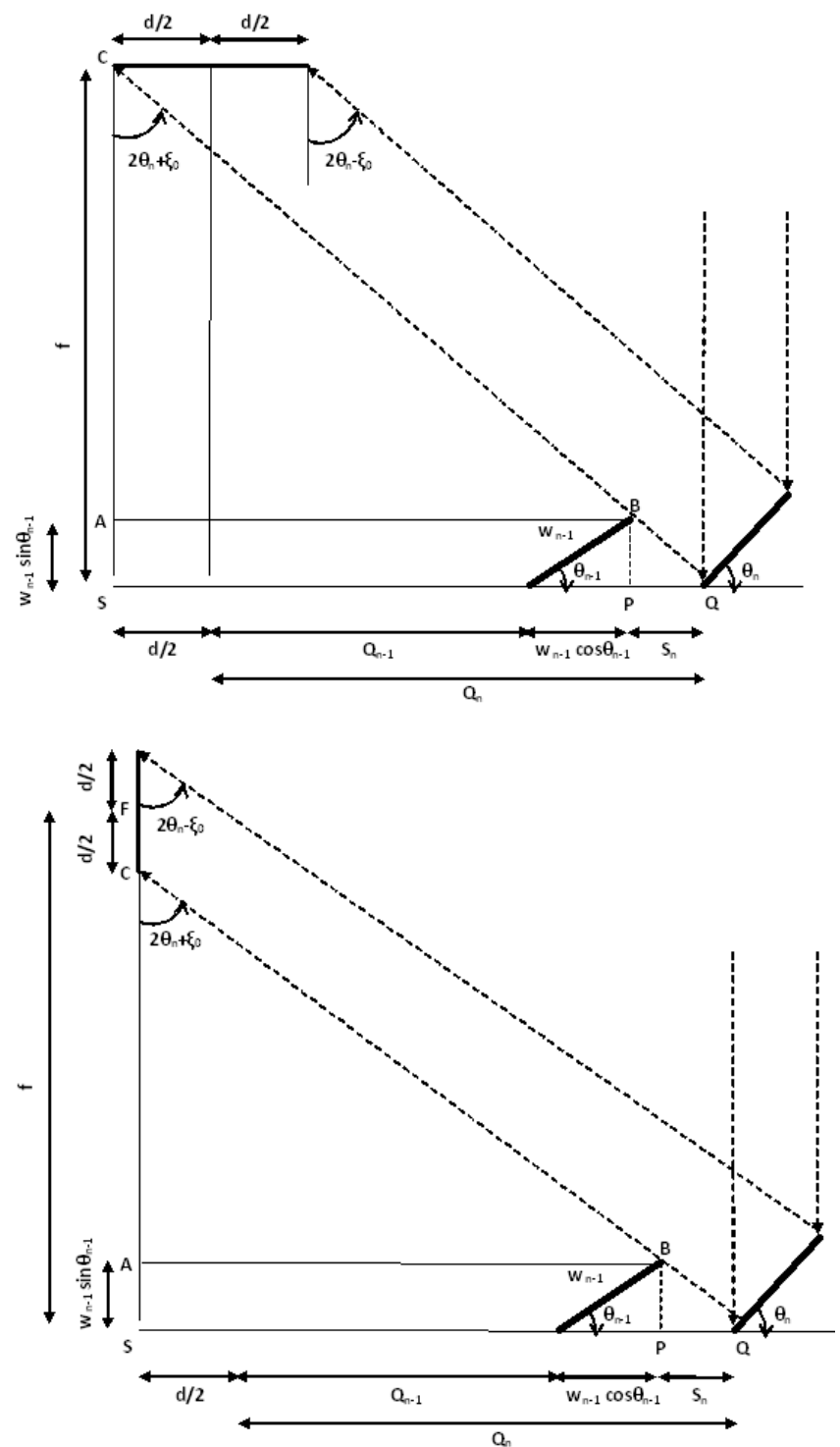

Figure 3 (a): Geometrical view of LFRCS system horizontal and vertical absorber with varying width reflector.

$\mathrm{d}=\frac{\left[\left(f-W_{n} \cdot \sin \theta_{\mathrm{n}}\right) \sec 2 \theta_{\mathrm{n}} \cdot \sin \xi_{0}\right]\left[\cos \left(2 \theta_{\mathrm{n}}-\xi_{0}\right)\right]+\left[W_{n} \cdot \cos \theta_{\mathrm{n}} \cdot \sec 2 \theta_{\mathrm{n}}\right]+[f \cdot \sec 2 \theta \cdot \sin \xi]}{\left[\cos \left(2 \theta_{0}+\xi_{0}\right)\right]}$ 
Rearranging equation (7) the width of the first mirror element be calculated from $W_{1}=\frac{d \sin \left(2 \theta_{1}+\xi_{0}\right) \cos \left(2 \theta_{1}-\xi_{0}\right)-\left(Q_{1}+d / 2\right) \sin \left(2 \xi_{0}\right)}{\sin \left(2 \theta_{1}+\xi_{0}\right) \cos \left(\theta_{1}-\xi_{0}\right)}$

The location of the second mirror element and its tilt with the concentrator plane are chosen such that the solar radiation reflected form it is not blocked by the first mirror element and finally produces an image on the surface of the absorber. Thus, a certain space is introduced between the first and second mirror element. The necessary shift for the second mirror element is given by

$$
S_{2}=\frac{\left(Q_{1}+W_{1} \cos \theta_{1}+d / 2\right) W_{1} \sin \theta_{1}}{f-W_{1} \sin \theta_{1}}
$$

The location of the second reflecting element and its tilt with concentrator plane is given by

$$
Q_{2}=\frac{Q_{1}\left(f-W_{1} \sin \theta_{1}\right)-d / 2 W_{1} \sin \theta_{1}}{f}-W_{1} \cos \theta_{1}
$$

and

$$
\theta_{2}=\frac{1}{2}\left[\tan ^{-1}\left(\frac{Q_{2}+d / 2}{f}\right)-\xi_{0}\right]
$$

The following are the generalized expressions for the shift $\left(S_{n}\right)$, location $\left(Q_{n}\right)$, tilt $\left(\theta_{n}\right)$, and the width $\left(W_{n}\right)$ are the parameters for the $\mathrm{n}^{\text {th }}$ mirror element of horizontal absorber is obtained by rearranging the above equations derived by $S_{n}=\frac{\left(Q_{n-1}+W_{n-1} \cos \theta_{n-1}+d / 2\right) W_{n-1} \sin \theta_{n-1}}{f-W_{n-1} \sin \theta_{n-1}}$

$$
Q_{n}=\frac{Q_{n+1}\left(f-W_{n} \sin \theta_{n}\right)-d / 2 W_{n} \sin \theta_{n}}{f}-W_{n} \cos \theta_{n}
$$

$\theta_{n}=\frac{1}{2}\left[\tan ^{-1}\left(\frac{Q_{n}+d / 2}{f}\right)-\xi_{0}\right]$

$W_{n}=\frac{d \sin \left(2 \theta_{n}+\xi_{0}\right) \cos \left(2 \theta_{n}-\xi_{0}\right)-\left(Q_{n}+d / 2\right) \sin 2 \xi_{0}}{\left(\sin \left(2 \theta_{n}+\xi_{0}\right)+\cos \left(\theta_{n}-\xi_{0}\right)\right)}$
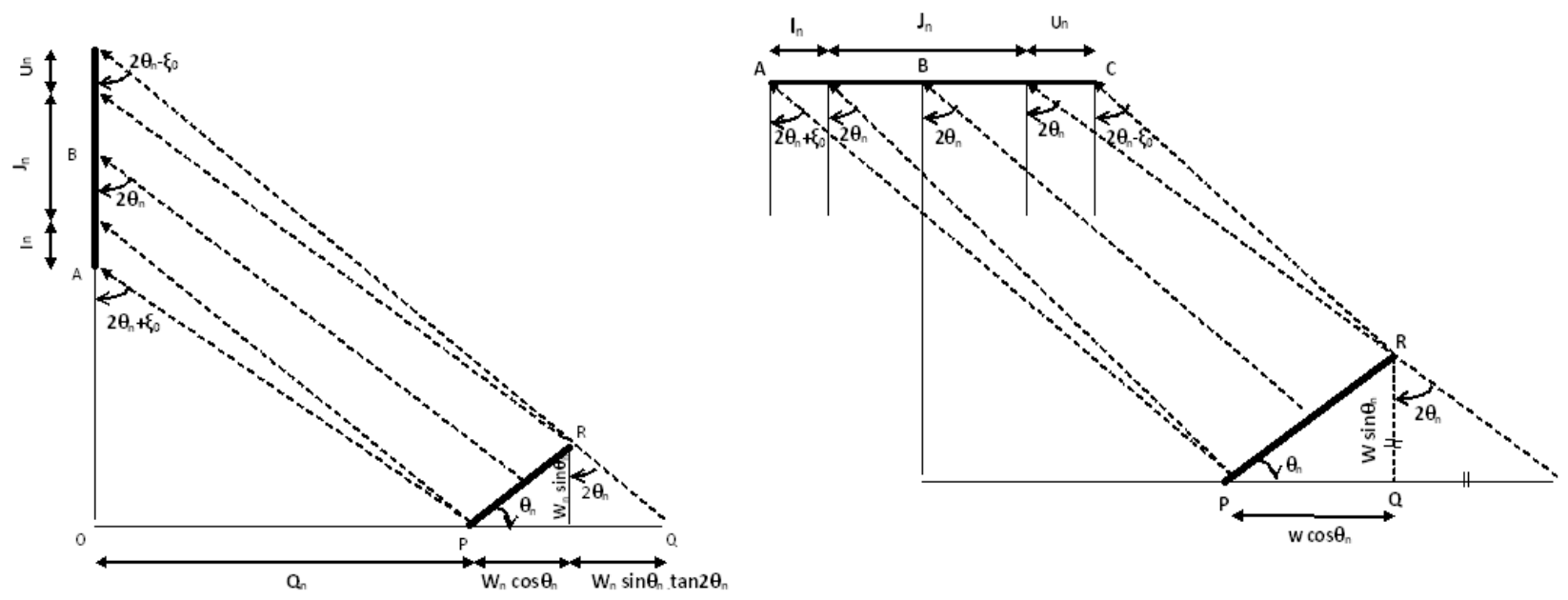

Figure 3 (b): Detailed Geometrical view of LFRCS system vertical and horizontal absorber with varying width reflector.

For the $\mathrm{n}^{\text {th }}$ mirror element of vertical absorbers,

$$
S_{n}=\frac{\left(Q_{n-1}+W_{n-1} \cos \theta_{n-1}\right) W_{n-1} \sin \theta_{n-1}}{f-W_{n-1} \sin \theta_{n-1}-d / 2}
$$




$$
\begin{aligned}
& Q_{n}=\frac{Q_{n+1}\left(f-W_{n} \sin \theta_{n}-d / 2\right)}{f-d / 2}-W_{n} \cos \theta_{n} \\
& \theta_{n}=\frac{1}{2}\left[\tan ^{-1}\left(\frac{Q_{n}}{f-d / 2}\right)-\xi_{0}\right] \\
& W_{n}=\frac{\left(d-Q_{n}\left(\cot \left(2 \theta_{n}-\xi_{0}\right)-\cot \left(2 \theta_{n}+\xi_{0}\right)\right)\right)}{\left(\sin \theta_{n}+\cos \theta_{n} \cot \left(2 \theta_{n}-\xi_{0}\right)\right)}
\end{aligned}
$$

\section{DISTRIBUTION OF LCR ON ABSORBER SURFACE FOR BOTH THE SYSTEMS}

In the analysis of LFRSC with vertical absorber [20] and LFRSC with horizontal absorber [18], it is assumed that the solar radiation reflected from each reflecting element is distributed uniformly over the width of the image the reflecting element produces on the absorber surface. The concentration at any point on the absorber is determined by summing up the contributions of all the reflecting elements. For a LFR employing reflecting elements of uniform and varying width, the contribution of any constituent reflecting element (say, $n^{\text {th }}$ ) to local concentration ratio (LCR) at any point within the image produced on the absorber by the reflecting element of absorber varying width on both horizontal and vertical is given by

$$
C I_{n}=\frac{W_{n} \cos \left(\theta_{n}\right)}{d}
$$

Since each constituent reflecting element illuminates the complete surface of the horizontal and vertical absorbers, total concentration $C R$ at any point on the surface of the absorber is given by

$$
C R=2 \sum_{n=1}^{k} C I_{n}
$$

In Fresnel concentrator tubular collector system, the reflector elements, reflect energy to different portions of the absorber and the concentrated flux from all the reflectors distributes large on the absorber. So, it is more meaningful to determine the total concentrated power reaching the absorber. Solar power $\left(P_{n}\right)$ that reached the absorber, which is contributed from the $n^{\text {th }}$ reflector, is given by,

$$
P_{n}=\rho I_{b} W_{n} \cos \theta_{n} L
$$

Where, $\rho$ is the reflectivity of the reflector and $I_{b}$ represents the intensity of beam radiation. Thus, the total concentrated power on the absorber due to the contribution from all the reflector elements is given by,

$$
C P=\left(2 \sum_{n=1}^{k} P_{n}\right)
$$

\section{RESULTS AND DISCUSSIONS}

In the present study, the area occupied by the Linear Fresnel Reflector Concentrating Solar (LFRCS) system is initially fixed and other system parameters like width of reflector plates, number of reflector plates, their tilt and relative positioning are determined using the expressions derived from the geometrical representation. Optimal design parameters are selected from the solutions which minimizes the material and land requirements. The design parameters of the LFRCS system are obtained by solving the expressions iteratively using MATLAB. In the iteration, the system parameters like width of reflector plates, number of reflector plates, and land area constraints are varied and the corresponding $C R$ is computed. The parameters utilized in the iteration that yields the maximum $C R$ are taken as the optimal design parameters. 


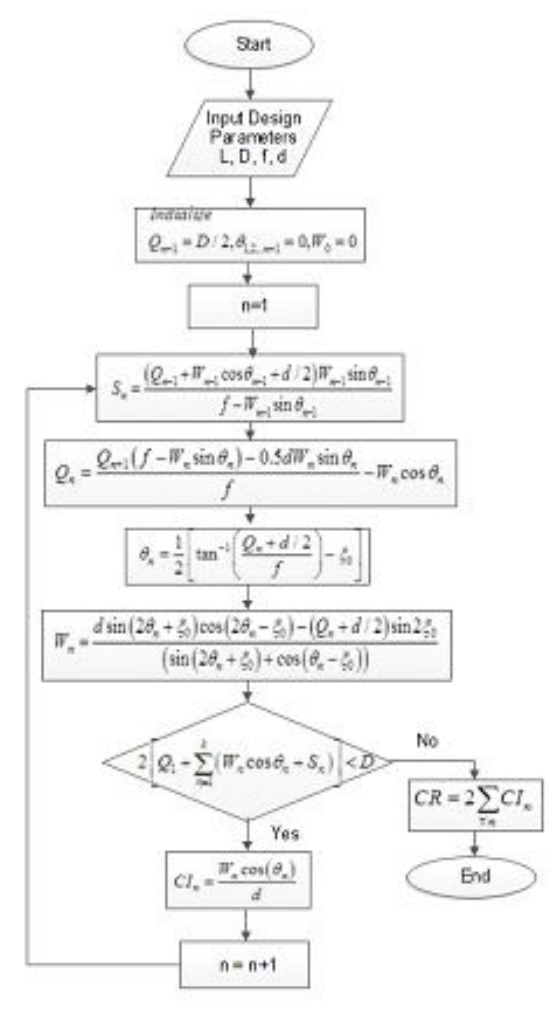

(a)

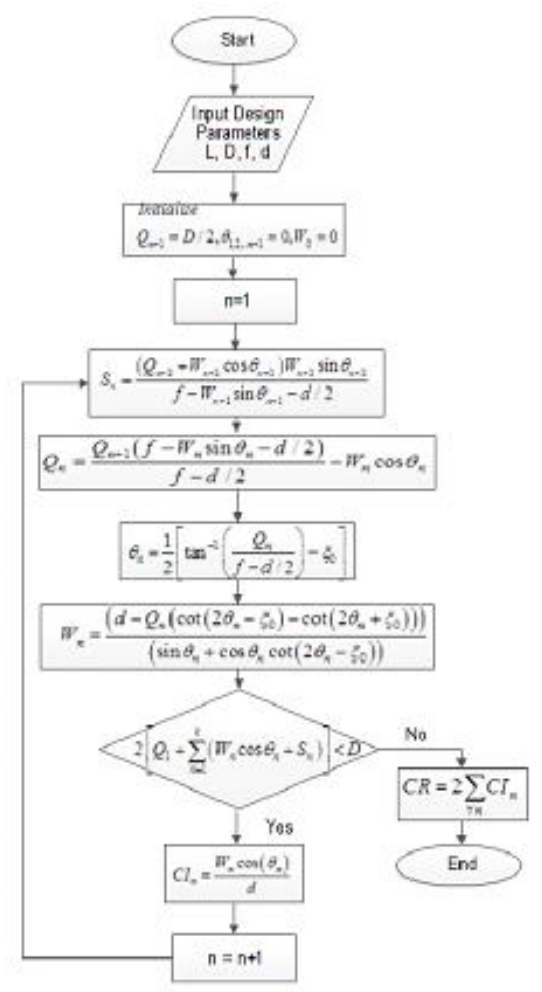

(b)

Figure 4 (a) (b): Flow chart for horizontal and vertical absorber system.

The schematic flow diagram showing the various calculations done for the horizontal absorber with varying width reflector in LFRC solar system and the vertical absorber with varying width reflector in LFRC solar system based on the design procedure are given in the Figure 4 (a) and Figure 4 (b) respectively

Two designs are considered for the present study, they are the horizontal system with varying width and vertical system with varying width. Let the aperture diameter, $D$ of the concentrator be $1.0 \mathrm{~m}$ for both the designs. For practical design, the required number mirror elements can be obtained as an integral multiple of the theoretical design. The proposed system is compared with the designs proposed in [20] and [18]. [20] Analyzed two flat vertical absorber LFRSC systems with mirror elements of varying and fixed widths and concluded that the CR of the vertical absorber with varying width is much higher than the design with fixed mirror elements. A CR of 8.9 is obtained for the vertical absorber placed at $0.5 \mathrm{~m}$ focal height and by setting the absorber diameter $d$ as $0.05 \mathrm{~m}$. Mathur considered two flat horizontal absorber LFRSC systems with mirror elements of varying and fixed widths, for same design parameters as considered above, a CR of 19.7 was achieved[18]. In both the above mentioned systems, the number of mirror elements is fixed arbitrarily and have claimed that the CR increases proportionally with respect to the total number of mirror elements. Practically, it is observed that even if the concentrated power appear to increase with number of reflectors [21], the extra-axial reflector beyond a particular number of reflector contribute little to the total concentrated power due to large cosine losses associated with them.

In both the above mentioned designs, the value of $C R$ decreases with an increase in the values of $d$ thus the careful selection of the diameter of the absorber for the LFRCS system is essential [20]. Also, consecutive mirror elements block the reflected radiation and a fraction of solar radiation incident over the aperture of the LFRC system is lost. The losses can be reduced and limited by providing an ample distance between consecutive mirror elements by introducing a shift $S$. This introduces an inherent constraint in the system design. The sum of the shifts provided between the mirror elements is directly proportional to the energy loss. In the proposed work, the shift $S$, tilt $\theta$ and width $W$ are computed by varying the absorber diameter $d$ from $0.01 \mathrm{~m}$ to $0.1 \mathrm{~m}$ and the focal height, $f$ from $0.1 \mathrm{~m}$ to $1 \mathrm{~m}$. The value of the absorber diameter $d$ and focal height that yields the maximum CR are finalized as the design parameters for conducting the experiments. In this scenario, the actual number of mirror elements that can 
be placed in the given design is obtained by following an iterative procedure. It is known that the aperture diameter $D$, of the concentrator can be expressed as

$$
D=2\left[Q_{1}+\sum_{n=1}^{k}\left(W_{n} \cos \theta_{n}+S_{n}\right)\right]
$$

Equation (36) can be modified to obtain the following condition to determine whether the aperture diameter D, is sufficient enough to include the next reflecting element.

$$
\text { if } 2\left[Q_{1}+\sum_{n=1}^{k}\left(W_{n} \cos \theta_{n}+S_{n}\right)\right]<D \quad \text { then add one reflecting element }
$$

else no space for additional reflecting element

The mirror element is added to the LFRCS system only when $2\left[Q_{1}+\sum_{n=1}^{k}\left(W_{n} \cos \theta_{n}+S_{n}\right)\right]<D$ for the given aperture diameter $D$. For practical values of $f$, an appropriate value of $D$ is taken to be around $2.0 \mathrm{~m}$. Using the condition in equation (25), the number of reflectors for the LFRCS system with horizontal and vertical absorbers are obtained as $N=29$ and $N=31$ respectively. This enables us to eliminate the extra- axial reflectors as well as utilize the aperture area for the given specification. It is well known that the maximum LCR is at the center and decreases proportionally with respect to the distance of the mirror element from the center of the absorber. This is due to the fact that the cones containing rays with angular deviation reflected from the mirror element placed near the center produce large spreading on the absorber. The spreading of the cones decreases as one move towards the rim of the concentrator from the center. Uniformity in the distribution of LCR can be achieved on the absorber surface by eliminating few mirror elements placed near the center of the concentrator plane, this may leave some area on the concentrator aperture unutilized [20]. In the proposed design, the aperture area is effectively utilized by placing the reflectors from the outer most mirror element (i.e. $\mathrm{n}^{\text {th }}$ ) towards the center thus the maximum aperture area is efficiently utilized.

\section{MODELING THE HORIZONTAL AND VERTICAL LFR SYSTEM WITH VARYING WIDTH REFLECTORS}

The performance of the horizontal and vertical absorber with varying width reflectors in terms of concentration ratio is analyzed. The aperture diameter for the experiments is fixed as $D=2 \mathrm{~m}$ and the absorber diameter " $d$ " is varied from 0.01 to $0.1 \mathrm{~m}$. For every absorber diameter, the focal height " $f$ " is diverged from 0.1 to $2.0 \mathrm{~m}$ and the respective concentration ratio is plotted. From the graph in figure 6(a), it is clear that the maximum concentration is obtained when the focal height is $f=0.9 \mathrm{~m}$ and absorber width is $d=0.05 \mathrm{~m}$. From the graph in

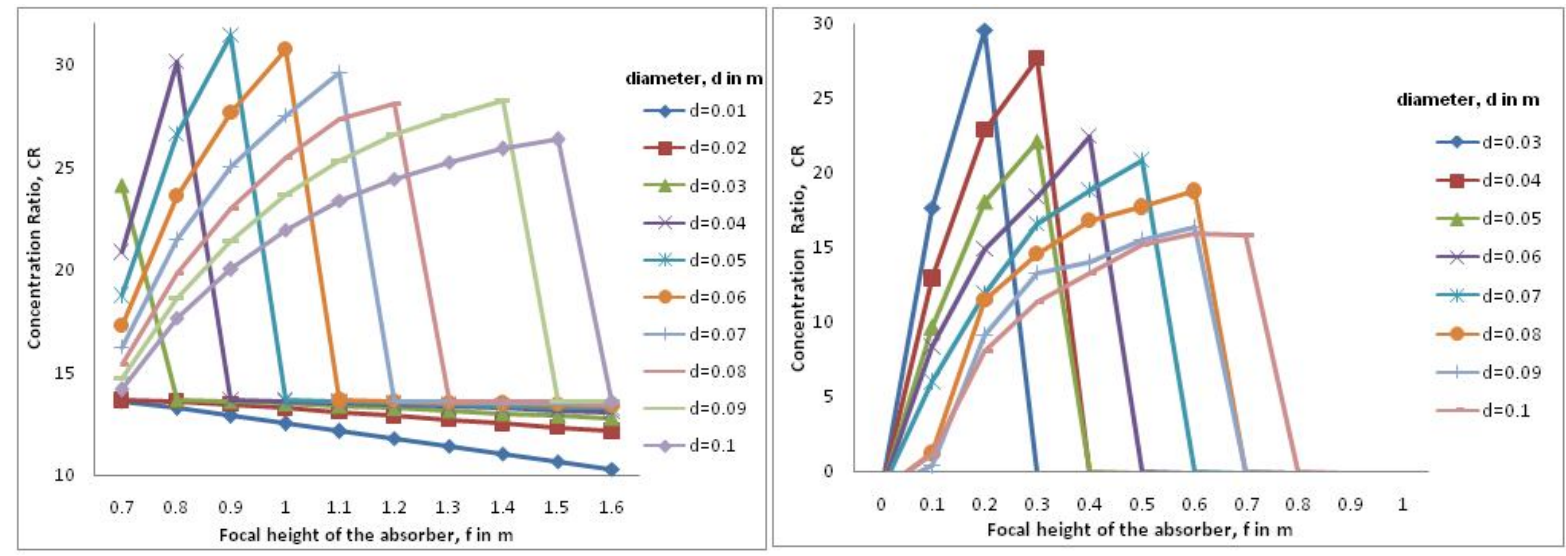

Figure 6(a,b): Performance analysis in terms of concentration ratio for horizontal and vertical absorber with varying width reflector system. 
Figure 6(b), it is clear that the maximum concentration is obtained when the focal height is $f=0.2 \mathrm{~m}$ and absorber width is $d=0.03 \mathrm{~m}$. In literature, the number of reflectors are varied arbitrarily for the given design parameters. In the proposed work, the maximum no of reflectors is solved computationally using equation (25) and for the horizontal design parameters the number of reflectors is determined as $N=29$. The system is modeled with the said parameters and the maximum concentration ratio is obtained as $C R=31.4$. For the vertical design parameters the number of reflectors is determined as $N=31$. The system is modeled with the said parameters and the maximum concentration ratio is obtained as $C R=29.5$.

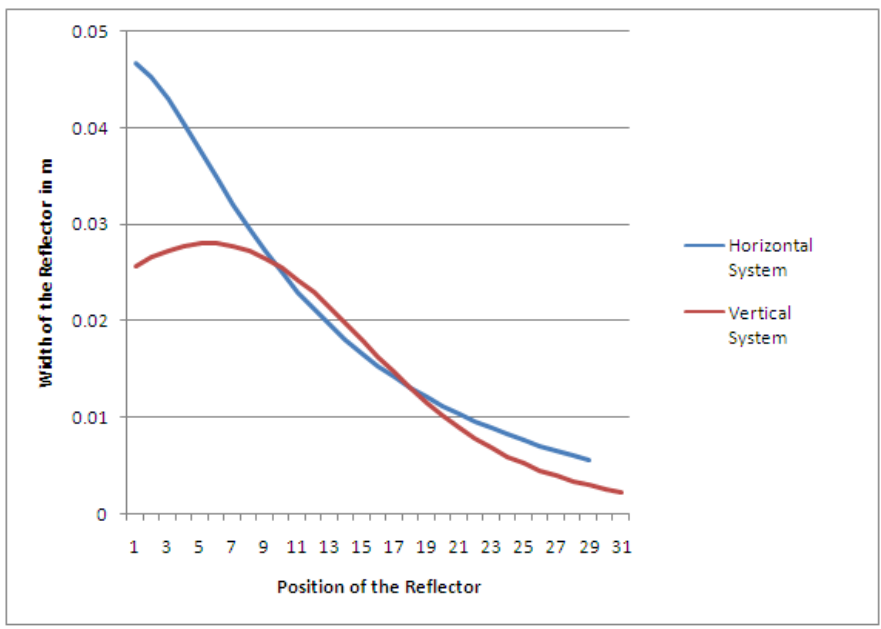

Figure 7: Width of the reflector vs. the position of the nth mirror element

Figure 7 shows the width of the $n^{\text {th }}$ reflector for all the $N$ reflectors obtained in both the designs. It also shows that the realization of the obtained reflector width is practically feasible. In this paper, mirror elements of varying width with different orientation of absorber are implemented and the results obtained are discussed in the modeling of the two different designs. From the experimental results, it can be observed that the absorber placed horizontally yields maximum concentration ratio and can be a preferred choice over the vertical absorber.

The error in the orientation of the mirror elements is modeled using the classical Gaussian distribution with zero mean $[22,23]$ and is expressed as

$$
p\left(\theta_{\varepsilon}\right)=\frac{1}{\sigma \sqrt{\pi / 2}} \exp \left[\left(-\frac{2 \theta_{\varepsilon}^{2}}{\sigma^{2}}\right)\right]
$$

Where $\theta_{\varepsilon}$ is the orientation error and $\sigma$ is the standard deviation of the Gaussian error. The unknown standard Deviation $\sigma$, is estimated using a penalized least squares estimation techniques proposed by Damien [24]. This method allows a fast smoothing of data in one and higher dimensions by means of the discrete cosine transform. Automatic choice of the amount of smoothing is carried out by minimizing the generalized cross-validation score. An iteratively weighted robust version of the algorithm is proposed to deal with occurrences of missing and outlying values which provides an efficient smoother for numerous applications in the area of data analysis.

Error correction can then be performed by subtracting the average error from all the measured orientation values. The average angular error $\overline{\theta_{\varepsilon}}$ or the mean of the Gaussian distribution can be determined as

$\overline{\theta_{\varepsilon}}=\int \theta_{\varepsilon} p\left(\theta_{\varepsilon}\right) d \theta_{\varepsilon} \quad=\frac{1}{\sigma \sqrt{\pi / 2}} \int_{0}^{\infty} \theta_{\varepsilon} \exp -\frac{2 \theta_{\varepsilon}{ }^{2}}{\sigma^{2}} d \theta_{\varepsilon}$

The integral in the above equation can be solved using the following identity in [25]

$$
\int_{0}^{\infty} x^{\nu-1} \exp \left(-\mu x^{p}\right)=\frac{1}{p} \mu^{\frac{-v}{p}} \Gamma\left(\frac{v}{p}\right)
$$


where the Gamma function $\Gamma(L)=\int_{0}^{\infty} t^{L-1} e^{-t} d t$. Thus, the solution of equation (28) is determined as

$$
\overline{\theta_{\varepsilon}}=\frac{1}{\sigma \sqrt{\pi / 2}} \times \frac{1}{2}\left(\frac{2}{\sigma^{2}}\right)^{-1} \Gamma(1)=\frac{\sigma^{2}}{4 \sigma \sqrt{\pi / 2}}=\frac{\sigma}{4 \sqrt{\pi / 2}}=\frac{\sigma}{5.0133}
$$

The orientation of the mirror elements obtained using equation (18) is corrected as

$$
\hat{\theta}_{n}=\theta_{n} \pm \overline{\theta_{\varepsilon}}
$$

Here, $\hat{\theta}_{n}$ is the orientation of the mirror elements after error correction. The effect of the orientation error on the concentration ratio of both the design is analyzed. In the first design, with horizontal reflectors of varying width the CR before and after error correction are obtained as 31.4418 and 31.4423 respectively. And for the second design, with vertical reflectors of varying width the CR before and after error correction are obtained as 29.5579 and 29.5580 respectively. The corresponding average orientation error (in degrees) in both the designs are $4.6224 \times 10^{-4}$ and $4.6375 \times 10^{-5}$. It can be verified that proposed theoretical system exercises a negligible orientation error on the concentration ratio of both the designs.

\section{CONCLUSION}

This paper analyzes the theoretical performance of LFR concentrating solar system with horizontal and vertical absorbers and with varying width reflectors by deriving the formulas for both the systems. From the results, it is evident that the LFR concentrating solar system with the horizontal absorber, varying width reflectors offers a better performance in terms of concentration on the surfaces of the absorber. The performance of the LFRCS system with vertical absorber varying width reflectors does not compare constructively with that of the horizontal absorber varying width design. In summary, the horizontal absorber with varying width LFRCS system produces better performance in terms of concentration ratio. This design can be used for solar thermal system particularly the horizontal system which can be used in pre heating feed water for boilers in steam generation and the concept of vertical system can be used in photovoltaic concentration system where uniform distribution of concentration on the solar photovoltaic cell is required, solar cells are exposed to the concentrated solar flux at the focus of concentrator, thus reducing the solar cells area and increasing the electrical output for a given capacity.

\begin{tabular}{|c|c|c|c|c|c|}
\hline Symbol & Description & Symbol & Description & Symbol & Description \\
\hline$Q_{n}$ & $\begin{array}{l}\text { Location of the } n^{\text {th }} \text { reflector } \\
\text { from the centre }(\mathrm{m})\end{array}$ & $\hat{\theta}_{n}$ & $\begin{array}{l}\text { orientation of the mirror elements after error } \\
\text { correction(degrees) }\end{array}$ & $C I_{n}$ & $\begin{array}{l}\text { Concentrated Power from } \\
n^{\text {th }} \text { reflector }(\mathrm{kW})\end{array}$ \\
\hline$P_{n}$ & $\begin{array}{l}\text { Power reflected from plate } n^{\text {th }} \\
\text { in }(\mathrm{kW})\end{array}$ & $\xi_{0}$ & $\begin{array}{l}\text { Half of the angular sub-tense of the sun at } \\
\text { any point on the earth }\left(=16^{\prime}\right)\end{array}$ & $\theta_{n}$ & $\begin{array}{l}\text { Tilt angle of } n^{\text {th }} \text { reflector } \\
\text { (degrees) }\end{array}$ \\
\hline$n, k$ & Number of reflector & $\rho$ & Reflectivity of the reflector & $I_{b}$ & Beam radiation $\left(\mathrm{W} / \mathrm{m}^{2}\right)$ \\
\hline$W_{n}$ & Width of the $n^{\text {th }}$ reflector (m) & $f$ & $\begin{array}{l}\text { Height of the absorber center from the base } \\
\text { (m) }\end{array}$ & $\sigma$ & $\begin{array}{l}\text { standard deviation of the } \\
\text { Gaussian error }\end{array}$ \\
\hline$d$ & Diameter of the absorber (m) & $D$ & Aperture diameter (m) & $\Gamma$ & Gamma function. \\
\hline$S_{n}$ & $\begin{array}{l}\text { Shift between the } n^{\text {th }} \text { reflector } \\
\text { (m) }\end{array}$ & $N$ & Number of reflectors & LFR & Linear Fresnel Reflector \\
\hline$L$ & Length of the absorber (m) & $C P$ & Total Concentrated Power $(\mathrm{kW})$ & LFRSC & $\begin{array}{l}\text { Linear Fresnel Reflector } \\
\text { solar concentrator }\end{array}$ \\
\hline$\overline{\theta_{\varepsilon}}$ & Average angular error(degrees) & $C R$ & Concentration Ratio & LCR & Local concentration ratio \\
\hline
\end{tabular}

VIII. SYMBOL AND ABBREVATIONS

\section{REFERENCES}

[1]. Canada. S, G. Cohen, R. Cable, D. Brosseau, and H. Price, Parabolic Trough Organic Rankine Cycle Solar Power Plant Conference Paper NREL/CP-550-37077 January (2005). 
[2]. Michael J Wagner, Simulation of direct steam power tower concentrated solar plant, ASME $6^{\text {th }}$ International conference on energy sustainability collocated with the ASME $10^{\text {th }}$ international conference on fuel cell science, engineering and technology, San diego, USA, 23-26 July, (2012).

[3]. Reynolds.D.J, M J Jance, M Behnia, and G L Morrison. An experimental and computational study of the heat loss characteristics of a trapezoidal cavity absorber, Solar Energy, 76(1-3):229-234, (2004).

[4]. Gabriel Morin, Hansjorg Lerchenmuller, Max Mertins, Markus Ewert, Mathias Fruth, Stefan Bockamp, Thomas Griestop, and Andreas Haberle. Plug-in Strategy for Market Introduction of Fresnel-Collectors. In SolarPACES, (2004).

[5]. . Panna Lal Singh, R.M.Sarviya and J.L Bhagoria, Thermal performance of linear fresnal reflecting solar concentrator with trapezoidal cavity absorbers, Applied Energy, Vol 87, Issue 2, pp 541-550,(2010).

[6]. Baum V. A., Aparasi R. R. and Garf B. A. High power Riaz M. R. A theory of concentrators of solar energy solar installations. Solar Energy 1, 6-13. (1976).

[7]. Francia G.Pilot plants of solar steam generation selective surface using bi-sublayer cermet film structures systems. Solar Energy 12, 51. (1968).

[8]. Riaz M. R.A theory of concentrators of solar energy on a central receiver for electric power generation. J. Eng. Power ASME 98, pp. 375-385, (1976).

[9]. Mathur. S.S, T. Kandpal, B. Negi, Optical design and concentration characteristics of linear Fresnel reflector solar concentrators - II. Mirror elements of equal width, Energy Conversion Management, Vol. 31, No.3, pp s221-232, (1991).

[10]. Martin Haagen, The potential of fresnal reflectors for process heat generation in the MENA region, Thesis submitted to faculty of electrical engineering and computer science, University of Kassel, (2012)

[11]. Manikumar. R , A. Valan Arasu \& S. Jayaraj ,Numerical simulation of a trapezoidal cavity absorber in the linear Fresnel reflector solar concentrator system International Journal of Green Energy, 08 Feb (2013).

[12]. Duffle. J.A and W. A. Beckman, Solar Engineering of Thermal Processes. John Wiley, New York, (1980).

[13]. Harry K. Charles, Jr., Solar photovoltaic energy systems, in Hand Book of Energy Technology and Economics, Chap. 16 (Edited by Robert A. Meyers). John Wiley, New York, (1983).

[14]. Mathur. S.S and T. C. Kandpal, Solar concentrators, in Reviews of Renewable Energy Resources, Chap. 5 (Edited by M. S. Sodha, S. S. Mathur and M. A. S. Malik). Wiley Eastern Ltd., New Delhi, (1984).

[15]. Marchi D.L, Design and construction of a one kilowatt concentrator photovoltaic system. Sandia Report SAND77-0909, Sandia Laboratories, Albuquerque, New Mexico, (1977).

[16]. David R. Mills and Graham L. Morrison, Advanced Fresnel Reflector Power plants - Performance and Generating Costs , Proceedings of Solar '97 - Australian and New Zealand Solar Energy Society, (2006).

[17]. Manikumar. R and A. Valan Arasu An Analytical and Experimental Study of the Linear Fresnel Reflector Solar Concentrator System, Distributed Generation and Alternative Energy Journal, Vol. 29, No. 2, (2014).

[18]. Mathur. S.S, B. S. Neci and T. C. Kandpal, Geometrical design and performance analysis of a linear Fresnel reflector solar concentrator with a flat horizontal absorber, Energy research, Vol. 14, 107-1 24. (1990).

[19]. Singh R.N., Mathur S.S., and Kandpal T.C Some geometrical design aspects of a linear Fresnel reflector solar concentrator, International journal of energy research, 4, 59- (1980).

[20]. Negi .B, T. Kandpal, S.S. Mathur, Designs and performance characteristics of a linear Fresnel reflector solar concentrator with a flat vertical absorber, Solar Wind Technology. 7, 379-392, (1990).

[21]. Manikumar. R and A.Valan Arasu, Design and theoretical performance analysis of linear Fresnel reflector solar concentrator with a tubular absorber, International Journal Renewable Energy Technology, Vol. 3, No. 3, (2012).

[22]. Badescu. V. Different tracking error distributions and their effects on the long-term performances of parabolic dish solar power systems. Int J Solar Energy. 14. 203-216. (1994).

[23]. Badescu, V. Theoretical derivation of heliostat tracking errors distribution, Sol. Energy, 82, pp. 1192 1197,doi:10.1016/j.solener.(2008)

[24]. Damien Garcia. Robust smoothing of gridded data in one and higher dimensions with missing values, computational Statistics and Data Analysis, 54. 1167-1178. (2010).

[25]. Gradhsteyn.S.I and I. M. Ryzhik, Table of Integrals, Series, and Products. New York: Academic. (1996) 